\title{
Simple Quality-of-Service Path First Protocol and Modeling Analysis*
}

\author{
Lin Shen, Mingwei Xu, Ke Xu, Yong Cui, Youjian Zhao \\ Department of Computer Science, Tsinghua University, Beijing, P.R.China, 100084 \\ \{shenlin, xmw, xuke, cy, zhaoyj\}@csnetl.cs.tsinghua.edu.cn
}

\begin{abstract}
QoS (Quality-of-Service) control will be one of the most important mechanisms in the next-generation Internet, where QoS routing (QoSR) is a promising solution. We propose a multi-constrained intradomain QoS routing protocol SQOSPF. The advantages of this protocol include easy implementation, multi-constrained QoS support, high-speed convergence and multiple QoSR algorithms support. Stochastic Petri Net is employed to model SQOSPF and analyze impacts of update threshold and routing holding time upon the load of networks and routers. Extensive simulations show that choosing appropriate update threshold and routing holding time can excessively reduce the extra load and keep routing performance at the same time.
\end{abstract}

Keywords-QoS routing, routing protocol, Petri Net, SQOSPF, performance analysis

\section{INTRODUCTION}

Current Internet, providing the best-effort service, cannot support QoS (Quality-of-Service) control. However, QoS control is one of the most important mechanisms in the nextgeneration Internet. And QoS routing is a promising solution. QoS routing (QoSR) seeks feasible paths subject to QoS requirements for the IP packets, and at the same time aims at achieving high efficiency in network resource utilization.

In order to support QoSR in the next-generation Internet, we need study QoS based on the connectionless hop-by-hop routing. That is to say, the QoS routing framework of the nextgeneration Internet has the characteristics (e.g. connectionless[1], hop-by-hop[2] and no resource reservation). To implement such a routing framework, it is necessary to study routing protocols that support these characteristics.

A multi-constrained intradomain QoS routing protocol SQOSPF (Simple Quality-of-Service Path First) is proposed in the paper. This protocol supports multiple QoS metrics (e.g. available bandwidth, delay, loss ratio and cost) and multiple core QoS routing algorithms. We also use the formal method (Stochastic Petri Net) to model and evaluate the protocol.

The rest of this paper is organized as the following. We give the specification of SQOSPF protocol in Section II. The SPN model is presented in Section III and simulation results are shown in Section IV. Finally, conclusions and future work appear in Section V.

\section{SQOSPF PROTOCOL}

\section{A. Outline of Protocol}

QOSPF is designed and implemented on the basis of simplified OSPF protocol. It only keeps two kinds of LSA in OSPF: Router LSA and External LSA[3][4]. The packets of QOSPF protocol are exchanged between adjacent intradomain routers. Router adopts the approach that combines time-driven and event-driven to broadcast protocol packets to adjacent routers. SQOSPF periodically acquires QoS metrics of links connected with local interfaces, and encapsulates these metrics into Router LSA to exchange with adjacent intradomain routers. By exchanging Router LSA, every router maintains network state information of AS (Autonomous System), including network topology and bi-direction QoS metrics of every links connected with interfaces of each intradomain router, and calculates the optimal paths to intradomain network address prefix by precomputation algorithms[7][8]. At the same time, the route computes the optimal paths to network address prefix of other ASs by integrating the received External LSA originated from a border router of an autonomous system with the optimal paths to that router. According to the optimal paths mentioned above, SQOSPF maintains the QoS routing table. When a connection request with QoS requirements arrives at a router, the router calculates a feasible path satisfying the QoS requirements for the request by on-demand algorithms[9][10]. When an IP packet arrives, the router seeks a feasible path in the QoS routing table according to the destination and the QoS requirements of the packet, and then forwards it. Therefore, SQOSPF can provide QoS guarantees to both connection-oriented mode and individual packet.

\section{B. Specification of Protocol}

We describe SQOSPF protocol according to the three cases that the router manages to deal with: (1) changes of local link state, (2) receipt of LSA (including Router LSA and External LSA), (3) receipt of redistributed routes. Before our description, we will introduce two definitions.

Definition 1. Optimal path

\footnotetext{
Supported by (1) the National Natural Science Foundation of China (NO. 90104002; No. 60373010); (2) the Key Project of Chinese Ministry of Education (NO.02004); (3) the National Major Basic Research Program (NO. 2003CB314801).
} 


\begin{tabular}{|c|c|c|c|}
\hline & 16 & & 4 \\
\hline Type & \# of Link & QoS Map & \\
\hline \multicolumn{4}{|c|}{ Advertising Router } \\
\hline \multicolumn{4}{|c|}{ Sequence Number } \\
\hline \multicolumn{4}{|c|}{ Link Address } \\
\hline \multicolumn{4}{|c|}{ Network Mask } \\
\hline QoS Mtr1 & QoS Mtr2 & QoS Mtr3 & QoS Mtr4 \\
\hline ....... & & & \\
\hline & .... & & \\
\hline
\end{tabular}

Figure 1. Router LSA

Given a path set $P_{<s, \triangleright}$ from source $s$ to destination $t$, a path $p \in P_{<s, t}$ is said to be an optimal path if there does not exist another path $p^{\prime} \in P_{<, \triangleright, \triangleright}$ such that $p^{\prime}$ is better than $p^{l}$.

As the "optimal "relationship of the paths in $P_{<\mathrm{s}, \triangleright}$ is partial order, that is to say, $P_{<s, \triangleright}$ is a partially ordered set, there may exist many optimal paths in $P_{<, t>}$.

\section{Definition 2. Relative variation ratio $(R V R)$}

Set $W$ as a certain QoS metric (such as available bandwidth, delay, etc.), the Relative Variation Ratio (RVR) of $W$ is defined as $\bar{W}=\frac{\mid W(\text { new })-W(\text { old }) \mid}{W(\text { old })}$, where $W($ new $)$ is the present value of $\mathrm{W}$, and $W($ old $)$ is the one at last update.

(1) Changes of local link state: SQOSPF periodically acquires QoS metrics of links connected with local interfaces, and then updates local router's information in the Intradomain Routers' Information Database (IRID) maintained locally. During updating, if RVR of a local link's QoS metric exceeds update threshold or a local link disconnects or resumes, Route LSA that represents local link state information will be broadcast to adjacent intradomain routers, and the recomputation of intradomain routes will be triggered.

(2) Receipt of LSA: when router receives a LSA sent by one of the adjacent intradomain routers, it will change to different processes according to the type of LSA.

(a) Router LSA: at first, if the sequence number of the LSA shows that the LSA is not out of date, it should be broadcast to neighbors, otherwise discarded. At the same time, IRID is updated according to the link state information encapsulated in the LSA. During updating, recomputation of intradomain routes will be triggered if the holding time is up and at least one of the following three conditions is satisfied: 1) The origin of the LSA is a new router of AS; 2) There are some pieces of information about addition and deletion of links in LSA; 3) RVR of a link's QoS metric exceeds the update threshold.

(b) External LSA: at first, if the sequence number of the LSA shows that the LSA is not out of date, it should be broadcast to neighbors, otherwise discarded. At the same time,
TABLE I

MEANING OF ROUTER LSA

\begin{tabular}{|c|l|}
\hline Type & Type of LSA, type of the Router LSA is 0x09. \\
\hline \# of Link & Number of the link information in the Router LSA. \\
\hline QoS Map & $\begin{array}{l}\text { QoS metrics mapping, and each bit corresponds to one } \\
\text { kind of QoS metric. }\end{array}$ \\
\hline Advertising Router & The identification of the origin of the LSA. \\
\hline Sequence Number & The sequence number of the Router LSA. \\
\hline Link Address & IP address of the interface connected with the link. \\
\hline Network Mask & Network mask of the interface. \\
\hline QoS Mtr.n & Values of QoS metrics that correspond to QoS Map. \\
\hline
\end{tabular}

the optimal paths to network address prefix of other ASs is calculated by integrating the LSA originated from a border router with the optimal paths to that router, and then the new routes are updated into the intradomain routing table.

(3) Receipt of redistributed routes: this case can only happen on the border routers that run interdomain routing protocol. The routes indicate the paths to the network address prefix of other ASs. When the case happens, SQOSPF updates the routes into the intradomain routing table and broadcasts External LSA composed of the redistributed routes to neighbors. Because of the restriction of IP packet's length, many External LSAs may be produced. The optimal paths with the same destination network address prefix must be encapsulated in an External LSA. If a LSA cannot hold all of the paths with the same destination prefix, the overflowing should be discarded directly.

\section{LSA Format}

Both kinds of LSA (i.e. Router LSA and External LSA) in SQOSPF are encapsulated in IP packets to exchange between routers. Router LSA is used to broadcast the QoS metrics of local links to the whole AS, the format of it and the meaning of each field are shown in Fig. 1 and Table I, respectively. The header of Router LSA is composed of five fields: Type, \# of Link, QoS Map, Advertising Router and Sequence Number, while Link Address, Network Mask and QoS Mtr.n compose link information. A Router LSA usually contains all the information of its origin's links.

External LSA is produced by border routers, and is responsible for broadcasting routes that are from border router to a certain network address prefix of other ASs to intradomain routers. The format of it and the meaning of each field are shown in Fig. 2 and Table II, respectively. The header of External LSA has two more fields than that of Router LSA: Destination Address and Network Mask, which are used to represent the destination network address prefix. Following the header is not link information but path information, which includes QoS metrics of each path. As there may exist many optimal paths to the same prefix, an External LSA will carry some pieces of optimal path information.

\section{SPN MODEL}

\footnotetext{
1 A path is better than another path iff each QoS metric of the former is better than that of the latter.
} 


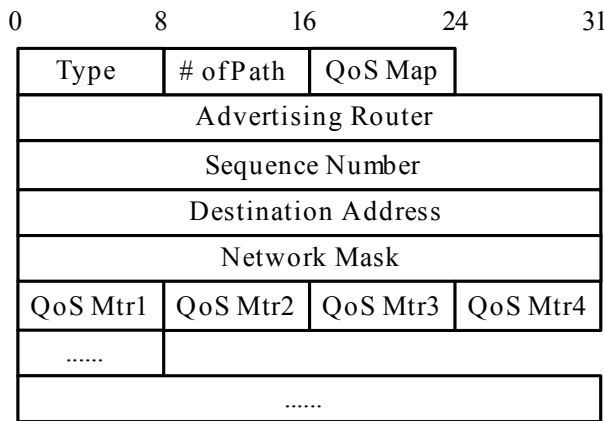

Figure 2. External LSA

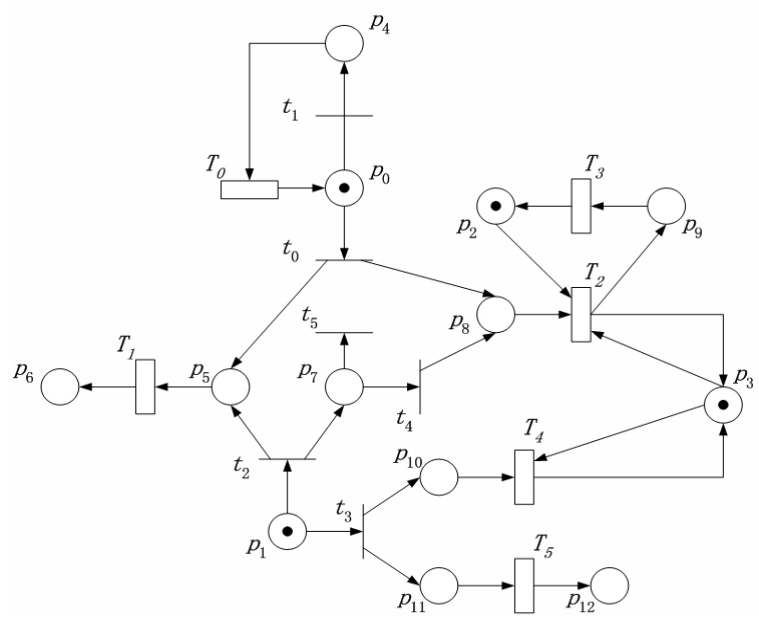

Figure 3. SPN model

Aiming at studying the framework and dynamic behavior of a system, Petri Net can strongly reflect the whole characteristics of the system. Stochastic Petri Net (SPN) introduces the notion of time, and adds a stochastic delay of a transition between "enabling" and "firing". As a result, the ability of simulation is strengthened[5]. Therefore, we employ SPN to model SQOSPF protocol.

The SPN model of the protocol is shown in Fig. 3. It is proposed on the basis of the alternation of the local link state and the disposal process of SQOSPF after receiving LSA. When it receives a redistributed interdomain route, a router can only take the route as an external LSA sent by itself.

Immediate transitions in the model are described as the following: transition t0 represents that the local link state has changed. In this condition, the routing recomputation and the broadcast of Router LSA can be triggered. Transition t0 is enabled under the circumstances that (1) a local link disconnects or resumes, or (2) RVR of a local link's QoS metric exceeds the update threshold. On the contrary, transition $\mathrm{t} 1$ represents that the local link state has changed but the routing recomputation and the broadcast of Router LSA should not be triggered. The unprocessed change is sent back to $\mathrm{p} 0$ through $\mathrm{p} 4$ and $\mathrm{T} 0$, which represents that the change will later be processed again by the router. When the router receives a Router LSA, t2 is enabled. So is t3 when the received is an External LSA. Intradomain routing
TABLE II

MEANING OF EXTERNAL LSA

\begin{tabular}{|c|l|}
\hline Type & Type of LSA, type of the External LSA is 0x0A. \\
\hline \# of Path & Number of the path information in the Router LSA. \\
\hline QoS Map & $\begin{array}{l}\text { QoS metrics mapping, and each bit corresponds to one } \\
\text { kind of QoS metric. }\end{array}$ \\
\hline Advertising Router & The identification of the origin of the LSA. \\
\hline Sequence Number & The sequence number of the Router LSA. \\
\hline $\begin{array}{c}\text { Destination } \\
\text { Address }\end{array}$ & $\begin{array}{l}\text { The IP address of the interface of the destination } \\
\text { router connected with the path. }\end{array}$ \\
\hline Network Mask & Network mask of the interface. \\
\hline QoS Mtr.n & Values of QoS metrics that correspond to QoS Map. \\
\hline
\end{tabular}

recomputation, represented by transition $t 4$, is enabled when recomputation is triggered after receiving Router LSA. Opposite to t4, transition $\mathrm{T} 5$ is enabled if no link state information can trigger the recomputation.

Timed transitions in the model is described as the following: transition T1 represents updating the local IRID and broadcasting Router LSA to neighbors, while T5 represents broadcasting External LSA to neighbors. The firing of T2 represents recomputation of intradomain routes according to the local IRID. When the processor is idle and the holding time is up, T2 can be enabled. T3 presents routing holding, the firing of which shows that the intradomain routes in routing table are guaranteed to be stable during the holding time. T4 represents recomputation of interdomain routes after External LSA is received. Its enabling also needs the processor.

The following describes places and tokens in the model: place $\mathrm{p} 0$ represents that change in local link state can be found after query. The tokens in p 0 reflect the change, and the number of them correlates with the alternation frequency. $\mathrm{p} 1$ represents receiving an SQOSPF protocol packet and determining its type. The tokens in $p 1$ represent two kinds of LSA. And the quantity of them correlates with not only the alternation frequency of the network state but also the scale of the whole AS. p2 represents the holding time is up, and intradomain routing recomputation can start. The token in $\mathrm{p} 2$ represents the computation can start right now, and there is only one token in $\mathrm{p} 2$. p3 represents acquiring processor. Its tokens represent processor resource, and its number is that of processors. p5 represents router starts updating IRID and broadcasting Router LSA, while p6 represents the router finishes. So is p11 to start broadcasting External LSA while p12 to finish. p7 represents whether recomputation can be triggered by the received Router LSA. p8 starts and $\mathrm{p} 9$ finishes intradomain routing recomputation, respectively. p10 represents that router receives an External LSA and recomputes interdomain routes.

Definition 3. Probability of not triggering recomputation $p_{r 1}$

In SPN model of SQOSPF, the enabling probability of t0 is called probability of not triggering recomputation $\boldsymbol{p}_{\boldsymbol{r} \boldsymbol{r}}$.

There are three random switchings in the model: 


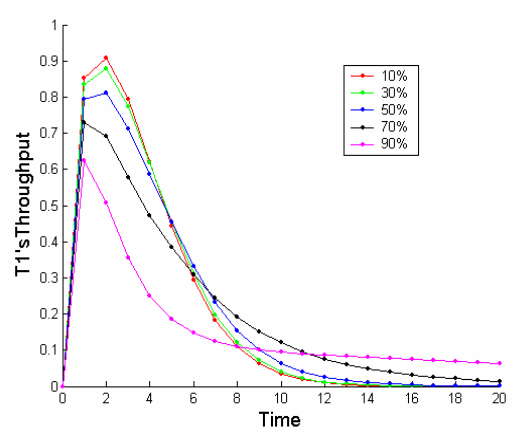

Figure 4. T1 's throughput

(1) If $M\left(p_{6}\right) \neq 0$ or $M\left(p_{12}\right) \neq 0$ :

$$
\begin{gathered}
\left\{\begin{array}{l}
P_{r}\left(t_{2}\right)=\frac{M\left(p_{6}\right)}{M\left(p_{6}\right)+M\left(p_{12}\right)} \\
P_{r}\left(t_{3}\right)=\frac{M\left(p_{12}\right)}{M\left(p_{6}\right)+M\left(p_{12}\right)}
\end{array}\right. \\
\text { If } M\left(p_{6}\right)=M\left(p_{12}\right)=0: P_{r}\left(t_{2}\right)=P_{r}\left(t_{3}\right)
\end{gathered}
$$

\section{PERFORMANCE ANALYSIS}

Spnp 6.0[11] is used to analyze the SPN model. The impact of update threshold and routing holding time upon network and routers' load is concerned. As the most serious problem of QoS routing at present is the great protocol and computation costs[6], our simulation aims at finding the factors that influence the costs, so that we can reduce certain costs and keep routing performance at the same time by adjusting these factors.

\section{A. Update Threshold}

In the AS that processes QoS routing by adopting SQOSPF, as the network state such as available bandwidth and delay changes frequently, update threshold is introduced to reduce the routing computation frequency and the impact of LSA upon network. When the RVR of QoS metrics of all the local links do not exceed the update threshold, routing recomputation will not be triggered and Router LSA will not be produced and broadcast. When the RVR of QoS metrics of all the links in the received Router LSA do not exceed the update threshold, routing recomputation will not also be triggered, but the received Router LSA will be broadcast to neighbors. However, the update threshold will lead to certain staleness, thus reduces routing satisfaction percentage. Therefore, how to choose update threshold so that our protocol can get the best performance is the goal of this simulation. The values selected for the model's parameters are shown in Table III and IV.

\section{Definition 4. QoS flow}

In an end-to-end QoS data communication, all of the packets in a single direction with same QoS requirements are made up of a QoS flow.
TABLE III

THE MEAN FIRING RATE OF TIME TRANSITIONS

\begin{tabular}{|c|c|}
\hline $\begin{array}{c}\text { Time } \\
\text { Transition }\end{array}$ & $\begin{array}{c}\text { Mean Firing } \\
\text { Rate }\end{array}$ \\
\hline $\mathrm{T} 0, \mathrm{~T} 1, \mathrm{~T} 5$ & 1.0 \\
\hline $\mathrm{T} 2, \mathrm{~T} 4$ & 0.1 \\
\hline
\end{tabular}

\section{Definition 5. Update accomplish time}

In the SPN model of SQOSPF, the time when the throughput of T1 is less than 0.00001 after reaching the peak is called the update accomplish time of Router LSA.

The update accomplish time of Router LSA reflects the update rate of the routes' information, and the update rate influences routing performance such as routing satisfaction percentage.

Fig. 4 shows the throughput of the transition $\mathrm{T} 1$ with different update threshold. The $\mathrm{x}$-axis is the simulation time. The curves from up to down correspond to the cases that pr1 is $10 \%, 30 \%, 50 \%, 70 \%$ and $90 \%$, respectively. We observe that the peak of T1's throughput is lower but the update accomplish time is longer with the rise of the update threshold. The reduction of the peak shows that the intensity of sending Router LSA decreases, which will reduce the network load caused by the Route LSA. However, because of the increase of the update accomplish time at the same time, the relative rate of the routing update to the arriving of QoS flow drops, and then the impact of the routes' staleness upon routing satisfaction percentage is enlarged.

Fig. 5 shows the throughput of the transition T2 with different mean firing rate of the transition T3 and update threshold. The $\mathrm{x}$-axis is the simulation time. The mean firing rate of T3 in the three figures is $0.0001,0.001$ and 0.01 , and the curves from up to down also correspond to the cases that pr1 is $10 \%, 30 \%, 50 \%, 70 \%$ and $90 \%$, respectively. We observe that the peak of T2's throughput decreases with the increase of the update threshold. As the throughput of $\mathrm{T} 2$ reflects the processors' load caused by the protocol, we can draw the conclusion that the load of processors decreases with the rise of the update threshold. This is because the increase of the update threshold reduces the computation frequency and then lowers the computation load of processors.

Considering the impact of the update threshold upon the load of network and processors and the update rate of routes' information together, we can keep a certain routing update rate without excessively increasing the load by choosing an appropriate update threshold, thus we can get a high routing satisfaction percentage.

\section{B. Routing Holding Time}

To avoid frequent updating of the routing table and routing jitter, we introduce routing holding time to limit the update frequency of the routing table. During the holding time, even if the intradomain routing recomputation is triggered for some reasons, the routes will not be calculated but kept unchanged. 


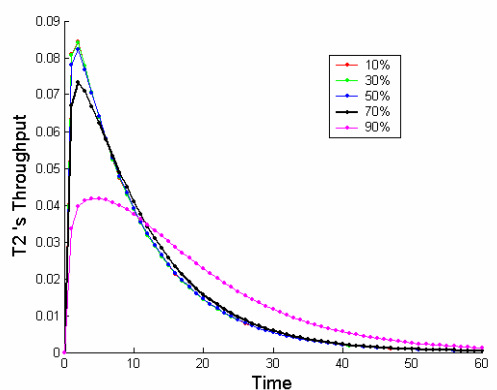

(a) $\mathrm{T} 3$ 's rate is 0.0001

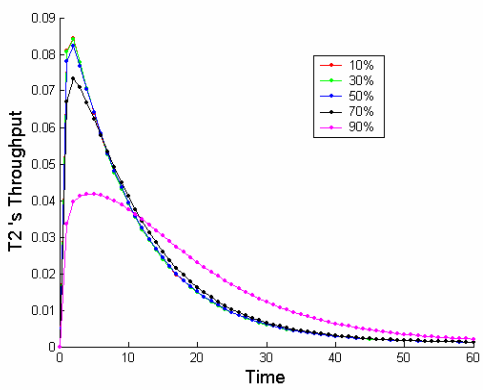

(b) $\mathrm{T} 3$ 's rate is 0.001

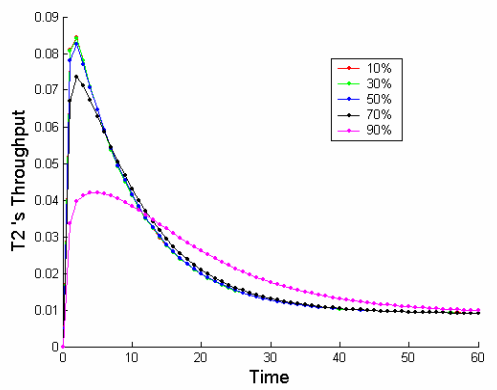

(c) $\mathrm{T} 3$ 's rate is 0.01

Figure 5. T2 's throughput

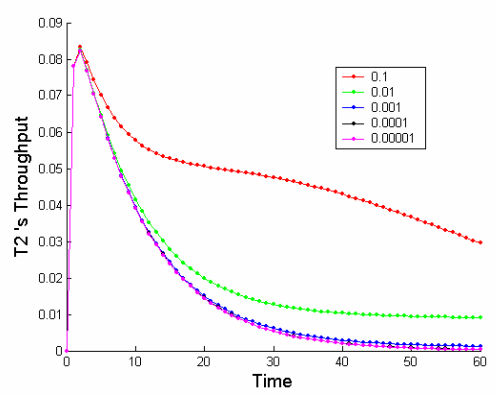

(a) $\boldsymbol{p}_{r 1}$ is $50 \%$

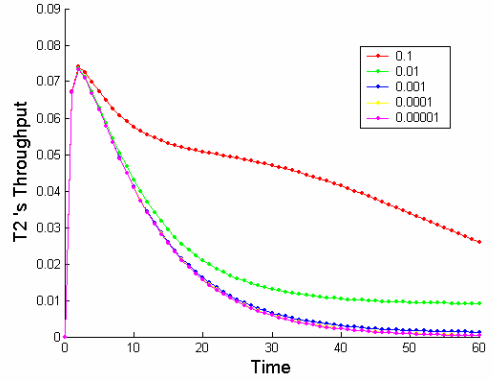

(b) $\boldsymbol{p}_{r 1}$ is $70 \%$

Figure 6. T2 's throughput

In the SPN model of SQOSPF, the mean firing rate of the transition T3 correlates with the holding time. In the following simulation, we will study the impact of routing holding time upon processors' load by modifying the mean firing rate of T3. The values for the model's parameters are also shown in Table III and IV.

Fig 6 shows the load of router's CPU with different holding time. The $\mathrm{x}$-axis is the simulation time. $\boldsymbol{p}_{r l}$ in the two figures is $50 \%$ and $70 \%$, respectively. T3's mean firing rate of the curves from up to down varies from 0.1 to 0.00001 . We observe that the CPU's load decreases obviously during the course of the reduction of the mean firing rate of T3 from 0.1 to 0.001 , but the change of the load is not obvious when the rate continue dropping from 0.001 . On the other hand, the increase of the routing holding time enlarges the routes' staleness. Therefore, CPU's load and routes' update rate must be considered together when holding time is configured. What is more, we observe that the peaks of the curves in $6(\mathrm{~b})$ are slightly lower than those of 6(a). This is because of the latter's less update threshold.

\section{CONCLUSION AND FUTURE WORK}

We propose an easy-implemented intradomain routing protocol SQOSPF that supports multi-constrained QoS metrics. The protocol is modeled on the basis of SPN and the SPN model is utilized to deeply analyze the performance of SQOSPF. We study the impact of update threshold and routing holding time upon network and routers' load. Extensive simulations show that the traffic and computational load can be decreased with the rise of update threshold and routing holding time. However, the routing staleness will be enlarged and routing performance will be decreased. Therefore, both load and routing performance should be considered together when the update threshold and routing holding time are configured.

It is our future work to study the interdomain routing protocols that support multi-constrained QoS.

\section{REFERENCES}

[1] Piet Van Mieghem, Hans De Neve, Fernando Kuipers, Hop-by-hop quality of service routing. Computer Networks 37(2001), pp. 407-423.

[2] Yong Cui, Jianping $\mathrm{Wu}, \mathrm{Ke} \mathrm{Xu}$. Multi-Constrained End-to-End Admission Control in Core-Stateless Networks, Procedings of 2004 International Conference on Information Networking (ICOIN'04), 2004.

[3] J. Moy, OSPF Version 2, April 1998, RFC 2328.

[4] G. Apostolopoulos, S. Kama, D. Williams, R. Guerin, A. Orda, T. Przygienda, QoS Routing Mechanisms and OSPF Extensions, August 1999, RFC 2676.

[5] Lin Chuang, Computer Network and Performance Evaluation of Computer System, Tsinghua University Press, Apr 2001.

[6] Yukinori Goto, Masataka Ohta, Keijiro Araki, Path QoS Collection for Stable Hop-by-Hop QoS Routing, Proceedings of INET'97, June 1997.

[7] Y. Cui, K. Xu, J.P. Wu, Precomputation for Multi-constrained QoS Routing in High-speed Networks, in Proceedings of the IEEE INFOCOM 2003, San Francisco, CA, April 2003.

[8] A. Orda and A. Sprintson, QoS routing: the precomputation perspective, IEEE INFOCOM'00, 2000, vol. 1, pp. 128-136.

[9] Y. Cui, J.P. Wu, K Xu, Z.C. Yu. Multi-constrained Routing Based on Simulated Annealing, IEEE 2003 International Conference on Communications (ICC).

[10] T. Korkmaz, M. Krunz, Multi-Constrained Optimal Path Selection, IEEE INFOCOM'01, 2001, pp. $834-843$, vol.2.

[11] http://www.ee.duke.edu/ chirel/research.html. 